\title{
Electrochemical machining of stainless steel microelements with ultrashort voltage pulses
}

Cite as: Appl. Phys. Lett. 79, 1721 (2001); https://doi.org/10.1063/1.1401783

Submitted: 08 June 2001. Accepted: 10 July 2001 . Published Online: 05 September 2001

Viola Kirchner, Laurent Cagnon, Rolf Schuster, and Gerhard Ertl

\section{ARTICLES YOU MAY BE INTERESTED IN}

Single-step electrochemical machining of complex nanostructures with ultrashort voltage pulses

Applied Physics Letters 82, 3327 (2003); https://doi.org/10.1063/1.1576499

Two-dimensional computational model for electrochemical micromachining with ultrashort voltage pulses

Applied Physics Letters 84, 3774 (2004); https://doi.org/10.1063/1.1738937

The state of the art in electrochemical machining process modeling and applications AIP Conference Proceedings 2017, 020029 (2018); https://doi.org/10.1063/1.5056292
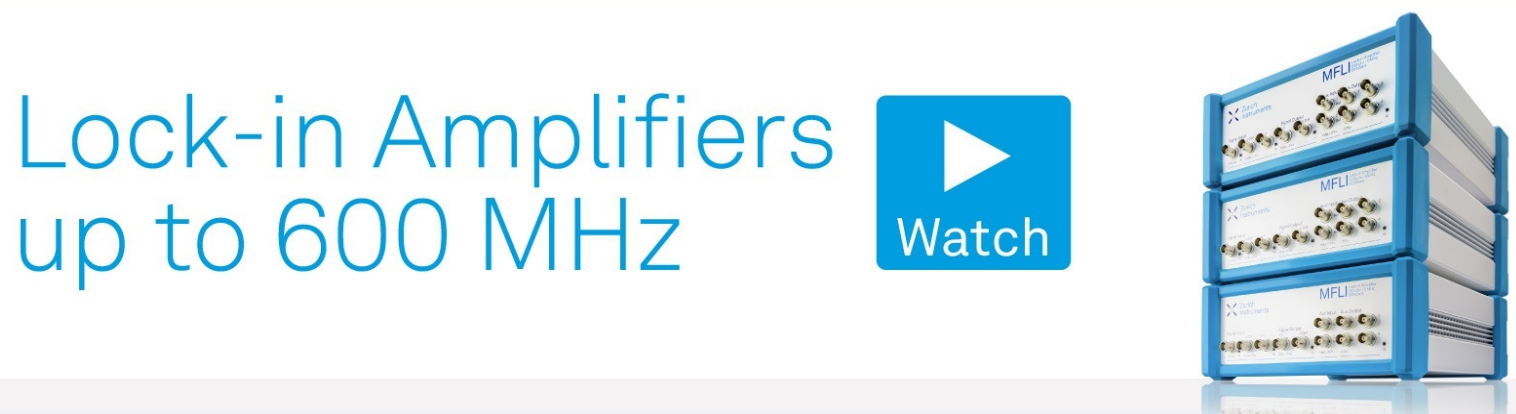


\title{
Electrochemical machining of stainless steel microelements with ultrashort voltage pulses
}

\author{
Viola Kirchner, Laurent Cagnon, Rolf Schuster, ${ }^{a)}$ and Gerhard Ertl \\ Fritz-Haber-Institut der Max-Planck-Gesellschaft, 14195 Berlin, Germany
}

(Received 8 June 2001; accepted for publication 10 July 2001)

\begin{abstract}
An electrochemical pulse technique enables the fabrication of three-dimensional microelements from stainless steel. The method is based on the application of ultrashort (nanosecond) voltage pulses, whereupon electrochemical reactions are locally confined with submicrometer precision. Employing properly shaped tool electrodes enables the machining of freestanding cantilevers or microstructures directly to a metal sheet. Due to gentle removal of the material, the grain structure of the material is revealed without any chemical or mechanical modifications. This is demonstrated by measuring the vibration frequency of a cantilever, and agrees well with the value derived from the bulk material properties. (C) 2001 American Institute of Physics. [DOI: 10.1063/1.1401783]
\end{abstract}

The fabrication of microdevices has become an important issue in modern technologies. ${ }^{1,2}$ Various techniques were developed to fabricate such structures from semiconductors, metals, and polymers. ${ }^{3}$ Most commonly, micromechanical devices are produced from silicon by lithographic techniques. These methods are, however, in principle restricted to two-dimensional (2D) structures. Three-dimensional (3D) structures can only be achieved in several steps, e.g., by a combination of lithographic techniques with anisotropic etching. The latter is, however, limited to single-crystalline materials. Recently, a combination of lithographic techniques with electrochemical metal deposition in the so-called LIGA process was used for the fabrication of small mechanical parts like gear wheels, but was again limited to structures with straight walls and homogeneous materials. ${ }^{4}$ [LIGA is the acronym for the German expressions for the main process steps: lithography (LI), electroforming (G), and molding (A)]. But for the micromachining of real construction materials like stainless steel, where the physical and chemical properties are tailored by the alloy composition, there are only few techniques available. These methods, however, suffer either from wear out of the tool, as in mechanical milling with miniature tools ${ }^{4}$ and microelectrical discharge machining, or from high cost and slow processing speed, as, e.g., with ion beam milling. ${ }^{5}$

We recently introduced an electrochemical method for three-dimensional machining of metals and semiconductors with submicrometer resolution and successfully demonstrated it for the structuring of copper and silicon. ${ }^{6,7}$ Here we apply this method to the machining of stainless steel as a real construction material with properties that are ideal for mechanical microdevices, e.g., chemical resistance and mechanical strength.

The method is based upon the application of short voltage pulses of nanosecond duration to a tool electrode, which leads to strong spatial confinement of electrochemical reactions with down to nanometer precision. Electrochemical reactions are driven by the potential drop across the electro-

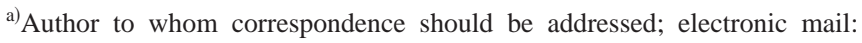
schuster@fhi-berlin.mpg.de
}

chemical double layer (DL) in front of the electrode surface. This DL consists of ions a few angstroms in front of the surface and the respective image charge in the metal electrode. It constitutes a plate capacitor with a very thin dielectric and a high capacity of the order of $10 \mu \mathrm{F} / \mathrm{cm}^{2}$. Upon application of a voltage step, this DL capacity is charged by current flowing through the electrolyte, which has a finite specific resistance. Thus, the charging time of the DL capacity becomes dependent upon the electrode separation. Therefore, with short enough voltage pulses the DL capacity is only significantly charged in electrode areas, where the current's path through the electrolyte is short enough, i.e., where the electrodes are in close proximity. Since electrochemical reaction rates are exponentially dependent on the voltage drop across the DL, electrochemical reactions, e.g., dissolution of material, are strongly confined to the charged regions of the DL.

By employing this electrochemical pulse method with a tiny tool electrode which is moved similar to that of a miniature milling cutter, three-dimensional structures with high aspect ratios can be etched into the material. Even undercuts and freestanding elements like the microcantilever shown in Fig. 1 can be easily fabricated in one step. The cantilever, which was machined within a few minutes directly into a 1.4301 stainless steel sheet, is $80 \mu \mathrm{m}$ long, $32 \mu \mathrm{m}$ wide, and $11 \mu \mathrm{m}$ thick, and hovers over a trough. To undercut the cantilever, we used a small loop made of a $10 \mu \mathrm{m}$ Pt wire, which was mounted perpendicularly in front of the workpiece. With a piezo-driven $x-y-z$ stage the loop was moved in three dimensions with respect to the workpiece. The loop and the steel foil, serving as electrodes, were immersed into an electrochemical cell with $3 \mathrm{M} \mathrm{HCl} / 6 \mathrm{M} \mathrm{HF}$ as electrolyte. In addition, the cell contained a $\mathrm{Pt}$ counterelectrode and a $\mathrm{Pd} / \mathrm{H}$ reference electrode to adjust the baseline potentials of the workpiece $\left(-300 \mathrm{mV}_{\mathrm{Pd} / \mathrm{H}}\right)$ and the tool $\left(200 \mathrm{mV}_{\mathrm{Pd} / \mathrm{H}}\right)$. A 1 $\mathrm{MHz}$ sequence of voltage pulses with $100 \mathrm{~ns}$ duration and 2 $\mathrm{V}$ amplitude was applied between the tool and the workpiece. The tool was first etched $30 \mu \mathrm{m}$ into the foil. Then the undercut was etched by moving the loop laterally $100 \mu \mathrm{m}$. On the flat top of the cantilever as well as on the surface of the sheet scratches from mechanical polishing were still vis- 

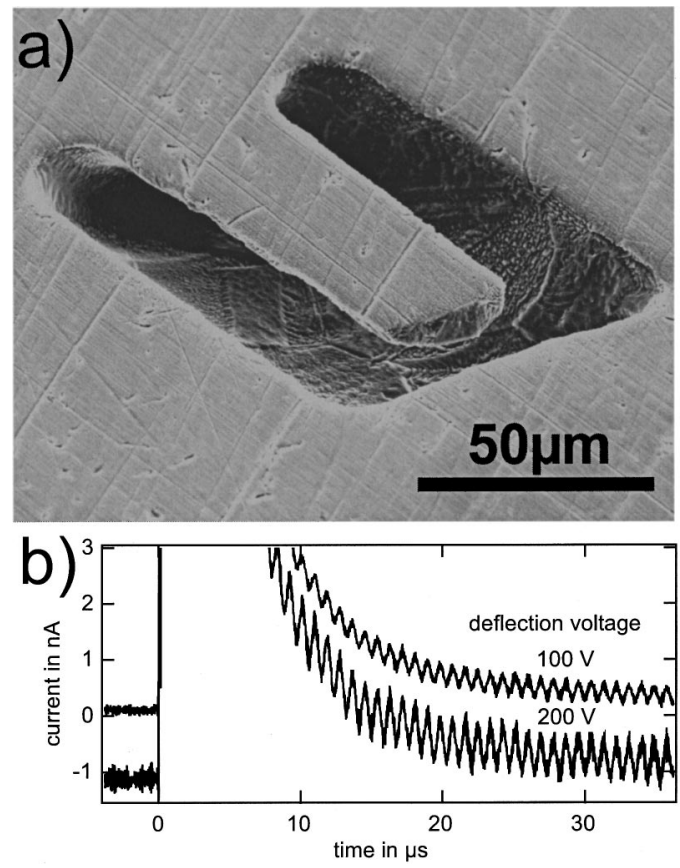

FIG. 1. (a) Scanning electron micrograph of a freestanding microcantilever machined into a stainless steel sheet by a sequence of $100 \mathrm{~ns}, 2 \mathrm{~V}$ pulses in $3 \mathrm{M} \mathrm{HCl} / 6 \mathrm{M}$ HF. The tool electrode was a tiny loop made from $10 \mu \mathrm{m} \mathrm{Pt}$ wire, which was etched perpendicularly into the surface followed by lateral movement. (b) Vibration of the microcantilever: After electrostatic deflection of the tongue by a dc voltage $(100$ or $200 \mathrm{~V})$ to an auxiliary electrode at $\mu \mathrm{m}$ distance, the voltage was switched down to $10 \mathrm{~V}$ at $t=0$, and the current oscillations across the varying auxiliary-electrode/cantilever capacitor were measured. Superimposed on the oscillatory signal is an exponentially decaying background caused by the recovery of the current amplifier.

ible, whereas inside the trough the grain structure of the stainless steel alloy could be recognized, indicating the unchanged structure and chemical composition of the metal sheet. We want to emphasize that the addition of both, $\mathrm{Cl}^{-}$ and $\mathrm{F}^{-}$ions to the solution is crucial for the micromachining of stainless steel. Upon oxidation a passivation layer of $\mathrm{Fe}$, $\mathrm{Cr}$, and $\mathrm{Ni}$ oxides forms on the surface, which inhibits further etching of the workpiece. This is the reason for the chemical resistance of stainless steel. $\mathrm{Cl}^{-}$and $\mathrm{F}^{-}$ions destabilize the oxide layer and thus assist in electrochemical dissolution. ${ }^{8}$ In the absence of $\mathrm{F}^{-}$we often observed the formation of a thick oxide layer, which eventually peels off the surface and renders micromachining impossible.

To demonstrate the mechanical properties of the cantilever, we measured its oscillation frequency in air by mechanical excitation of vibrations. The cantilever was electrostatically deflected by applying a dc voltage ( 100 or $200 \mathrm{~V}$ ) to a flattened $50 \mu \mathrm{m}$ diam wire, whose face is placed above the cantilever at $\mu \mathrm{m}$ distance. Switching the voltage down to 10 $\mathrm{V}$ within about $100 \mathrm{~ns}$ resulted in oscillations of the tongue which were measurable as current oscillations across the varying capacitor, formed by the cantilever and the deflection electrode [Fig. 1(b)]. The amplitude of the capacitive current is proportional to the initial deflection of the cantilever, i.e., the applied voltage step. Superimposed on the oscillating current signal is an exponentially decaying background caused by the relatively slow recovery of the current measuring amplifier after the high voltage step.

The oscillation frequency measured amounts to 1.1 $\mathrm{MHz}$. From the dimensions of the tongue, determined from

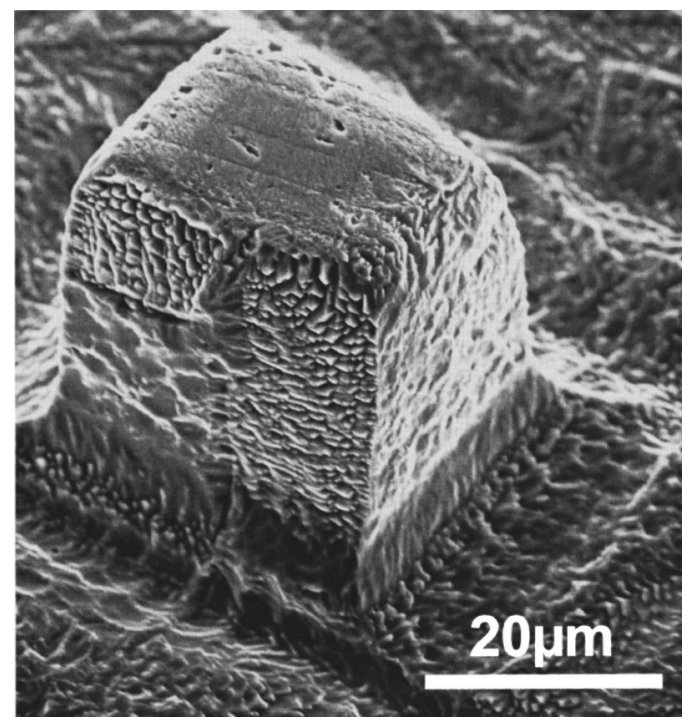

FIG. 2. SEM image of a 1.4301 stainless steel microcube, electrochemically machined with $100 \mathrm{~ns}$, and $2 \mathrm{~V}$ pulses. The faces show microfacets, varying with the different orientations of the steel grains, and sharp grain boundaries between crystals with different orientations.

scanning electron microscope (SEM) images, we calculated a vibration frequency of $0.7 \mathrm{MHz}$ employing the bulk elastic modulus of 1.4301 stainless steel of $2 \times 10^{11} \mathrm{~N} / \mathrm{m}^{2} .{ }^{9}$ Considering the power-law dependence of the oscillation frequency on the dimensions of the tongue, the calculated frequency is in reasonable agreement with the experimental one. Although the vibration measurement was performed in air, only weak damping of the oscillations was observed. The damping decrement is estimated from longer measurements (data not shown) to be $5 \times 10^{3} \mathrm{l} / \mathrm{s}$, which results in a $Q$ factor of the order of $10^{3}$. The vibrating cantilever provides a very sensitive microbalance, e.g., for measuring even single monolayers of adsorbates: Changing the mass of the cantilever in Fig. 1 by the adsorption of one monolayer of $\mathrm{CO}$ is estimated to decrease the oscillation frequency by about 4 Hz. Thinner cantilevers would exhibit even higher sensitivity. Such cantilevers could be machined from metal single crystals with very specific adsorption properties, which opens new avenues for sensor applications.

The good agreement between the theoretical and the measured oscillation frequency can be attributed to the fact that during electrochemical micromachining neither melting of material nor mechanical deformation occurs. The single crystallites of the steel are cut without changing the texture, which is demonstrated in more detail in Fig. 2 which shows a scanning electron micrograph of a microcube machined into a 1.4301 steel foil with a cylindrical $50 \mu \mathrm{m}$ wire with a flattened face. Upon applying a $1 \mathrm{MHz}$ sequence of $100 \mathrm{~ns}$ voltage pulses $(2 \mathrm{~V})$, the wire was fed $30 \mu \mathrm{m}$ into the workpiece vertically, followed by lateral movement along a rectangular path under similar conditions as those for the fabrication of the cantilever. The faces of the cube exhibit microfacets typical of anisotropic etching, which varies with the different crystal orientation of the steel grains. A sharp grain boundary to a crystal with a different orientation can be seen in the lower left part on the front face of the cube. The texture of the bulk material is exposed in 3D, which opens up interesting aspects for investigation, e.g., of depth profiles 
of the grain structure without applying mechanical polishing. Note the sharpness of the perpendicular edges of the microcube, which is well below $1 \mu \mathrm{m}$, although the width of the gap between the tool and the surface in this experiment was about $5 \mu \mathrm{m}$.

As mentioned above, electrochemical micromachining is based on the finite charging time of the electrochemical double layer capacity on the electrode surfaces, due to the finite electrolyte resistance. Therefore, the machining precision, i.e., the degree of confinement of the electrochemical reactions, is directly related to the pulse length. In Ref. 6 we demonstrated for the machining of $\mathrm{Cu}$ that the spatial resolution is linearly dependent on the pulse length. Experiments with stainless steel and shorter pulses down to $10 \mathrm{~ns}$ duration revealed the same behavior. The ultimate spatial resolution we achieved in these experiments was well below $1 \mu \mathrm{m}$. Extrapolated from these results, the application of $100 \mathrm{ps}$ pulses should achieve resolutions in the $10 \mathrm{~nm}$ range. The useful application of such resolutions for the machining of real devices would, however, require tools of similar precision.

In summary, we have demonstrated that electrochemical micromachining with ultrashort voltage pulses is well suited for technical applications. Freestanding microstructures from real construction materials like stainless steel can be fabricated in a single step with high machining rates. Future ap- plications may include a combination with standard lithographic techniques to produce insolating layers for the implementation of complete electrode arrays for use as microelectromechanical devices, sensors, or actuators.

The authors want to thank G. Heyne, V. Platschkowski, W. Däumel, and M. Kock for support during the experiments. Discussions with P. Allongue and K. Weil are gratefully acknowledged.

${ }^{1}$ H. G. Craighead, Science 290, 1532 (2000).

${ }^{2}$ G. L. Timp, Nanotechnology (Springer, New York, 1999).

${ }^{3}$ Handbook of Microlithography, Micromachining, and Microfabrication, edited by P. Rai-Choudhury (SPIE, Bellingham, WA, 1997), Vols. 1 and 2.

${ }^{4}$ C. R. Friedrich, R. Warrington, W. Bacher, W. Bauer, P. J. Coane, J. Göttert, T. Hannemann, J. Hausselt, M. Heckele, R. Knitter, J. Mohr, V. Piotter, H.-J. Ritzhaupt-Kleissl, and R. Ruprecht, in Handbook of Microlithography, Micromachining, and Microfabrication, edited by P. RaiChoudhury (SPIE, Bellingham, WA, 1997), Vol. 2, p. 299.

${ }^{5}$ D. K. Stewart and J. J. D. Casey, in Handbook of Microlithography, Micromachining, and Microfabrication, edited by P. Rai-Choudbury (SPIE, Bellingham, WA, 1997), Vol. 2, p. 153.

${ }^{6}$ R. Schuster, V. Kirchner, P. Allongue, and G. Ertl, Science 289, 98 (2000).

${ }^{7}$ V. Kirchner, X. Xia, and R. Schuster, Acc. Chem. Res. 34, 371 (2001).

${ }^{8} \mathrm{H}$. Kaesche, Metallic Corrosion Principles of Physical Chemistry and Current Problems (National Association of Corrosion Engineers, Houston, 1985).

${ }^{9}$ W. T. Thomson, Theory of Vibration (George Allen and Unwin, London, 1986). 